\title{
Philosophiques
}

François Duchesneau, Genèse de la théorie cellulaire, Montréal-Paris, Bellarmin-Vrin, collection Analytiques, 1987, $388 \mathrm{p}$.

\section{Maurice Gagnon}

Volume 16, numéro 2, automne 1989

URI : https://id.erudit.org/iderudit/027087ar

DOI : https://doi.org/10.7202/027087ar

Aller au sommaire du numéro

Éditeur(s)

Société de philosophie du Québec

\section{ISSN}

0316-2923 (imprimé)

1492-1391 (numérique)

Découvrir la revue

\section{Citer cet article}

Gagnon, M. (1989). François Duchesneau, Genèse de la théorie cellulaire, Montréal-Paris, Bellarmin-Vrin, collection Analytiques, 1987, 388 p.

Philosophiques, 16(2), 395-404. https://doi.org/10.7202/027087ar d'utilisation que vous pouvez consulter en ligne.

https://apropos.erudit.org/fr/usagers/politique-dutilisation/ 


\section{ÉTUDE CRITIQUE}

FRANÇOIS DUCHESNEAU, Genèse de la théorie cellulaire, Montréal-Paris, Bellarmin-Vrin, collection Analytiques, 1987, 388p.

par Maurice Gagnon

Des raisons historiques et épistémologiques expliquent le choix du sujet en titre. D'une part, on ne conçoit plus aujourd'hui l'histoire des sciences comme une simple accumulation additive de connaissances; on considère aussi et surtout les mutations des modes de théorisation et des cadres conceptuels. D'autre part, le modèle proposé par Kuhn n'est pas suffisamment complexe pour permettre d'entrer dans les détails de ces mutations, spécialement en histoire de la biologie, où les modèles empiriques sont plus complexes et moins mathématiques, les théories moins précises qu'en physique. Enfin, dans un ouvrage antérieur (La physiologie des lumières. Empirisme, modèles et théories, La Haye, Martinus Nijhoff, 1982), l'A a voulu « retracer les modes de théorisation qui délimitent progressivement un champ de recherche, celui des fonctions vitales dans leur rapport aux éléments structuraux de l'organisme » (p. 10). Cette recherche a révélé une succession de modèles physiologiques qui aboutissent à la conception cellulaire. Le présent ouvrage poursuit l'analyse entreprise par le précédent : il examine les mutations subies par la conception cellulaire pendant ses débuts, soit au cours du $19^{\mathrm{e}}$ siècle. L'A. considère d'emblée la théorie cellulaire comme une théorie unifiée proclamant que les cellules sont les constituants fondamentaux de tous les organismes, qu'elles ont une structure commune, et qu'elles sont la source de toutes les fonctions vitales (p. 345). L'objectif épistémologique qu'il s'est fixé est le suivant : «établir le profil synchronique et diachronique d'un programme de recherche dans ses phases initiales, phases que l'on peut qualifier d'《embryonnaire», si l'on tient compte de phases d'évolution à l'état " explicite ", qui le mèneront jusqu'à la biologie directement contemporaine $»($ p. 14).

L'A. rejette le falsificationnisme poppérien pour deux raisons : «l'absence d'argument menant à l'énoncé d'hypothèses susceptibles de résistance croissante à l'infirmation empirique (test expérimental), et le caractère paradoxal d'une progression rationnelle par négations successives » (p. 14). Il adopte plutôt le schéma de Lakatos: l'activité scientifique est faite de programmes de recherche donnant lieu à des théories successives dont chacune explique des 
faits plus nombreux et plus variés que la précédente, et transforme les instances infirmatives de cette dernière en instances corroboratives. Mais il demeure critique par rapport à ce schéma: d'abord, l'heuristique négative d'une théorie (l'ensemble de ses présupposés de type métaphysique et méthodologique) n'est pas complètement indépendante de son heuristique positive (recettes méthodologiques qui permettent de développer la recherche), puisque la première suscite et motive la seconde, est jugée selon la fécondité de cette dernière, et doit donc être relativisée en conséquence; ensuite, la théorie cellulaire ne correspond pas entièrement à ce que Lakatos appelle un programme, soit « un édifice logique servant à articuler des concepts relatifs à un ordre circonscrit de phénomènes» (p. 16), mais elle combine des phénomènes très hétérogènes, appartenant à plusieurs domaines d'étude, et vaut « surtout comme structure argumentative susceptible de révéler l'unité fonctionnelle " (ibid.) de ces phénomènes; et enfin, la théorie cellulaire n'est pas un ensemble d'explications causales et de lois formant un programme articulé de recherches, mais «un simple cadre d'analyse de phénomènes complexes associant des causalités plus élémentaires de divers types » (p. 17).

La première partie du volume retrace l'évolution de la théorie cellulaire de Dutrochet à Raspail et Schwann dans une atmosphère antivitaliste, de 1824 à 1853 . Dutrochet voit dans des «vésicules celluleuses ou tubuleuses » (p. 22) mises en contact par force d'adhésion ou d'agglutination et pourvues chacune d'une paroi distincte, les éléments de tout tissu organique. Grâce à un jeu d'analogies fondées sur des comparaisons entre animaux différents, entre animaux et végétaux et entre cellules et cristaux, il explique par la forme, la position, la nature chimique des cellules, par les substances qu'elles secrètent, de même que par la nature osmotique et autres propriétés de leurs parois, les structures différentes des tissus et des organes, la motilité, la nutrition et la croissance, bref toutes les fonctions physiologiques et vitales. La reproduction cellulaire est pour lui un phénomène endogène, grâce à des corpuscules contenus dans la membrane ou paroi de la cellule-mère et pouvant se développer en elle.

Sur le plan épistémologique, Dutrochet voit la science comme inductive et visant à établir la corrélation des faits et leurs liaisons mutuelles, et comme activité de synthèse dégageant ce que les faits ont de commun et de fondamental. Elle use de modèles mathématiques universels pour transformer en « faits d'intuition intellectuelle " (p. 41) les théories, qui sont des théorèmes articulant rationnellement l'expérience (ibid.). Il refuse les hypothèses comme "conjectures sans validation" (ibid.), mais admet, à l'intérieur des théories, le recours à la probabilité «comme moyen de direction pour l'investigation » (ibid.), de même que le recours à des « postulats » concernant les "causes inapercevables" (ibid.) des faits physiques perçus, postulats ultérieurement validés par les faits qu'ils ont permis de détecter. Il écarte le vitalisme aussi bien comme principe ontologique que comme ensemble de concepts régulateurs déterminant un champ de phénomènes autres que physico-chimiques : les concepts vitalistes n'ont pas pour lui de référent dans 
les faits expérimentaux, et il pose donc que les lois physiologiques sont en principe réductibles à des lois physico-chimiques. Il rejette en conséquence l'idée d'une distinction essentielle entre vivant et non-vivant: la croissance par intussusception, supposément propre au vivant, est en continuité avec l'aggrégation, qui est une de ses phases; et le vivant est, tout autant que le non-vivant, déterminé de l'extérieur.

Pour Raspail, les cellules (vésicules élémentaires) sont des êtres organisés, véritables laboratoires capables d'attirer en elles, de l'extérieur, tout ce qu'il leur faut pour se reproduire et croître. Elles sont produites par une organisation, c'est-à-dire une cristallisation sui generis qui, par superposition de molécules, en fait non pas des entités aux formes anguleuses comme les cristaux, mais des entités de forme vésiculaire (sacs) qui constituent ensuite les organes et les embryons en croissant et en se modifiant selon des formes et des structures déterminées et variables selon les cas. Raspail tient que les explications chimique, anatomique et physiologique sont nécessaires et complémentaires pour expliquer l'organisation vitale, cette dernière étant cependant irréductible à une simple superposition. Les éléments et mécanismes physico-chimiques sont donc subordonnés à la description des structures organiques élémentaires et à leurs caractéristiques fonctionnelles et doivent montrer comment ces structures et fonctions sont effectivement réalisées. Raspail admet donc le principe d'une émergence de l'organisation vitale, mais n'affirme ni ne rejette le réductionnisme physico-chimique. La réduction effective suppose, dit-il, une explication de l'organisation à partir de phénomènes élémentaires, mais aussi une fonction d'intégration des mécanismes chimiques et physiologiques, possiblement physico-chimique elle aussi, et que nous ne connaissons pas présentement. Il compare cette fonction à une inconnue algébrique sans toutefois refuser de l'appeler «force vitale». Il s'agit d'un vitalisme méthodologique, d'un ensemble de concepts régulateurs des divers aspects de l'expérience. Les principaux concepts de Raspail sur l'explication des fonctions vitales sont les suivants: d'abord celle de vésicule imperforée dont la paroi est faite de vésicules plus petites, et ainsi de suite jusqu'à un corpuscule premier (comme chez Dutrochet), aspiration et expiration, et transformation des gaz et des liquides par la vésicule ; cristallisation vésiculaire, c'est-à-dire formation des vésicules par des éléments chimiques simples sur le modèle de la formation des cristaux.

Une théorie est pour Raspail un énoncé d'application très générale contenant le moins d'exceptions possible, expression réelle ou hypothétique d'une loi dont on reconnaît l'existence sans pouvoir en préciser tous les éléments. Elle met en forme des éléments empiriques épars en introduisant entre eux des relations qui anticipent des lois et qui sont fondées sur des analogies. La meilleure théorie est celle qui suffit à expliquer les faits actuellement connus, et elle sert de guide à l'observation.

Schwann reprend pour l'essentiel plusieurs idées de Dutrochet et de Raspail sur la cellule: elle est l'unité de base de tous les vivants; son processus de développement est le prototype de toute activité physiologique ; 
elle exclut toute idée de forces vitales non physico-chimiques; son rôle métabolique est réductible à des processus physico-chimiques; la structure cellulaire peut se transformer, ce qui explique la diversité fonctionnelle des systèmes vivants. Il conserve en outre le concept d'organisme comme unité intégrative et intégrée, dotée d'une marge d'autonomie par rapport aux déterminismes du milieu, sans toutefois adopcer l'architectonisme de Müller, dont nous parlerons plus loin. La continuité de Dutrochet et Raspail à Schwann n'est cependant pas rectiligne et totale: Schwann puise aussi à d'autres sources ou formule ses propres doctrines. Il affirme la distinction noyau-protoplasme-membrane, soutient que le développement de la cellule se fait du nucléole au noyau puis du noyau à la membrane, à partir d'un cytoblastème (germe de cellule) relevant des «milieux intérieurs » de l'organisme et dont la matrice est située au niveau du derme, voit dans le protoplasme une série de couches superposées successivement de l'intérieur vers l'extérieur au cours d'une épigenèse, et explique le développement de l'embryon en termes de développement et reproduction cellulaire.

Ces dernières remarques amènent l'A à étudier les idées du maître de Schwann, Müller. Pour ce dernier, la recherche empirique doit être subordonnée à une causalité téléologique: un agent vital, ou force vitale, conçu sans analogie psychologique, est nécessaire pour réaliser les composés organiques vitaux en combinaisons tétradiques ou triadiques, alors que les combinaisons sont exclusivement binaires dans le monde inorganique. Cet agent vital coordonne les opérations matérielles organiques et génétiques. Müller croit trouver des structures déjà complexes dans l'embryon et refuse d'utiliser l'hypothèse globulaire (cellulaire) pour expliquer le processus organogénétique : l'individualité organique commande la détermination des propriétés et des forces en jeu dans l'activité organique, et donc aussi dans la formation du futur individu. Cette théorie de la force vitale n'est pas purement spéculative et a priori, car son concept est déterminé par des analogies empiriques disponibles. La nutrition, la croissance et la régénération supposent un nisus formativus, une force régulatrice des processus métaboliques, une force qui intègre une structure virtuelle, conforme à l'archétype de l'organisme, et qui coordonne en direction d'un organisme complètement formé tous les processus bio-physico-chimiques, dont chacun obéit à des lois spécifiques. Müller intègre dans sa physiologie l'embryologie de von Baer, qui est une théorie physiologique des capacités vitales émergentes dues à la forme et à la combinaison des matières organiques. L'embryon se développe, selon von Baer, en vertu d'une combinaison organique propre à chaque espèce, ceci supposant une organisation fonctionnelle qui est déjà virtuellement existante et qui gouverne un processus de structuration au cours duquel sont d'abord réunis les matériaux nécessaires (l'ovule, par exemple); viennent ensuite la formation différenciative des organes à partir de ces derniers, puis l'agencement des organes entre eux, le tout selon une série de stuctures successives emboîtées, chacune intégrant la précédente. Il existe une vis formativa, héritage des dispositions fonctionnelles propres à chaque espèce, et les mécanismes cellulaires ne jouent ici qu'un rôle secondaire. L'ordre synthétique 
des choses, prédéterminé, gouverne l'ontogenèse et guide donc l'analyse empirique. Il y a donc pour Müller un «projet organique " qui incarne une conception holiste et architectonique de l'organisme exerçant les fonctions vitales, et Müller combine l'affirmation de ce principe avec une recherche empirique des structures, processus et lois qui servent d'instruments à ce projet pour la réalisation de ses fins. Un examen du programme de recherches de Schwann s'avère nécessaire pour voir jusqu'à quel point ce dernier s'écarte d'une telle vision des choses. C'est l'objet de la troisième partie du volume.

Réductionniste d'emblée, Schwann le devient davantage au contact de Schleiden. Empiriste, partisan de l'induction guidée par des maximes heuristiques qui ne font aucune place au holisme et à l'architectonisme à la MüllerBaer, Schleiden voit dans les cellules l'unité vivante fondamentale, du moins chez les végétaux, et dans leurs regroupements selon diverses lois l'explication de l'organisme, qui n'en est qu'une résultante, et non un projet virtuellement présent à l'origine dans les matériaux. Schwann combine tout cela avec des principes d'analogie, empruntés à Dutrochet et Raspail, entre les structures réciproques des végétaux et des animaux.

Les Mikroskopiscbe Untersuchungen de Schwann analysent d'abord les structures fondamentales de l'organisme, et établissent en premier lieu une homologie structurale et une analogie de disposition fonctionnelle entre des cellules de la corde dorsale et des cartilages d'une part, et d'autre part des cellules végétales: la cellule est donc universelle dans sa structure et comme élément fondamental du vivant. Il montre ensuite l'universalité de la séquence morphologique cellulaire chez les tissus animaux, fait appel à la théorie du cytoblastème pour expliquer la formation et la structure initiale de l'ouf, puis parle ensuite de sa modification par différenciations successives, caractérisées chacune par la genèse de tissus nouveaux faits de cellules dites secondaires, c'est-à-dire formées par une coalescence de cellules primaires où ces dernières perdent leur autonomie, mais où le tout conserve les fonctions de cellule. Les cellules maintiennent pendant tout ce processus, le métabolisme de base qui leur est commun, c'est-à-dire croissance et assimilationdésassimilation.

Vient ensuite une synthèse où, en premier lieu, Schwann systématise et synthétise les données empiriques utilisées dans l'analyse précédente. Il formule dans des lois les conditions matérielles de formation des cellules et de leurs échanges physiologiques, bâtissant ainsi une représentation de la construction et du fonctionnement de l'organisme, de même que de ses échanges avec d'autres organismes et avec le milieu non organique. La cellule est présentée comme l'archétype de l'organisme, ce qui est un programme de recherche plutôt qu'un résultat, et qui est incompatible avec toute idée de force vitale et de projet architectonique, de totalité virtuellement présente dans un matériel initial. En second lieu, Schwann crée l'hypothèse d'une série d'analogies entre cristaux et cellules en ce qui concerne leur structure et leur formation; cette hypothèse, elle aussi incompatible avec le vitalisme, a pour fonction de guider les recherches à venir sur les conditions matérielles de la 
cytogenèse et sur la formation complète de l'organisme par les cellules. Les cellules y sont présentées comme des cristaux imbibables, capables d'intussusception et de pouvoirs métaboliques particuliers, avec comme arrièreplan le cytoblastème d'origine. Ce modèle semble d'ailleurs aller moins loin que le programme déjà entrepris de décrire l'organisme comme formé par combinaison de cellules transformées à des degrés divers, mais gardant toujours les mêmes fonctions essentielles.

Les interprétations diverses de la théorie de Schwann par Müller, Kölliker et Remak font l'objet de la quatrième partie de l'ouvrage. En 1838 , Müller attribue aux cellules, telles que décrites par Schwann, des formations pathologiques (cancer). Il maintient cependant la conception, rejetée par Schwann, d'un organisme comme totalité architectonique, les cellules morbides rompant cette harmonie. En 1840, dans son Handbuch der Physiologie des Menchen, le métabolisme cellulaire explique les fonctions vitales, mais la séquence morphogénétique qui dérive les cellules des fluides formateurs intra-organiques est expliquée par un principe de vie, une idée directrice gouvernant le tout vers un organisme total. Il prête ce pouvoir à certaines cellules.

De son côté, Kölliker fait l'analyse histologique de la cellule, qui se forme selon lui autour d'un noyau. Il conserve l'hypothèse du cytoblastème mais abandonne peu à peu l'analogie cristalline, soutenant que la cellule est hétérogène à toute structure inorganique. Il décrit la reproduction cellulaire comme une scission et une formation endogène, alors que Schwann parlait de formation exogène. La cellule a pour lui une structure dualiste dont les éléments sont le protoplasme et le noyau, et il voit en elle le cadre irréductible des processus physico-chimiques sous-jacents à la vie. Le protoplasme est substrat des échanges moléculaires, alors que le noyau déclenche, coordonne et contrôle les processus liés à ces échanges. Cela permet à la cellule d'être une individualité vivante irréductible et autonome, capable de croître et de se reproduire. Elle est en outre capable d'interagir avec son milieu ambiant : elle réagit à son état et contribue à le former et le transformer.

Remak s'intéresse surtout à la reproduction cellulaire, qui est pour lui une division interne de la cellule à partir du noyau. Il récuse les thèses schwanniennes de la formation libre des noyaux à partir du cytoblastème et des analogies entre cristaux et cellules, car elles lui paraissent inadéquates par rapport à l'idée d'une organisation vitale élémentaire: la réplication de la cellule par division suppose une individualité organique. Le noyau et ses parties jouent dans la cellule un rôle architectonique, et il y a donc un schème de progression du centre à la périphérie; mais il faut aussi admettre un conditionnement du centre par la périphérie, en raison des altérations méraboliques subies par le protoplasme. Pour la première fois une même représentation, soit la division cellulaire, intègre à la fois la formation de la cellule et ses modifications internes.

La cinquième partie traite du modèle cellulaire physiologique de Virchow. Ce dernier voulait fonder une physiologie pathologique et étudia dans ce but 
les phénomènes inflammatoires, cas typiques de processus physiologiques perturbés. Il repéra alors des dégénérescences typiques de cellules, notamment des séquences dégénératives dans la reproduction cellulaire, et par conséquent remit en cause la genèse blastémique. Utilisant avec profit les nouvelles idées de Remak (Omnis cellula e cellula), il formula un nouveau concept de cellule comme lieu des échanges moléculaires de l'organisme, et comme totalité vivante fondamentale, l'organisme étant une totalité dérivée. Le noyau joue un rôle architectonique sur toutes les activités de nutrition de la cellule, qui permettent de reproduire cette dernière et de la maintenir en vie. Chaque cellule a une zone d'intervention (territoire cellulaire) sur les autres cellules et sur le milieu interstitiel. À partir de cela, Virchow offre une explication de la formation des tissus nouveaux, et des phénomènes pathogènes comme l'inflammation : ils sont dus à des modifications dans la nutrition des cellules. Ces vues ont influencé Kölliker, qui a cependant tenté de les couler dans un langage et une vision plus mécanistes, et elles ont été reprises par Claude Bernard, pour qui les cellules produisent un milieu intérieur d'autant plus autonome par rapport aux déterminismes extérieurs qu'il est plus différencié.

Dans une conclusion où il résume les principales étapes de la conception cellulaire, l'A. se livre à quelques considérations épistémologiques qui nous intéressent particulièrement ici. Dans les premières phases de la genèse de la théorie cellulaire, il remarque la recherche d'une structure élémentaire commune à tous les vivants, par-delà le nombre et la variété des fonctions vitales et des espèces animales et végétales, et donc aussi d'une représentation unique et d'une explication commune pour les phénomènes vitaux. En ceci la théorie cellulaire est d'abord un schéma à préciser ultérieurement par des concepts et des lois, et elle ne manque alors pas de parenté avec la théorie atomique en physique, bien que dans cette dernière discipline les lois intégrées à la théorie visent aussi à décrire des mécanismes aboutissant à la production de phénomènes déterminés, donc à la prévision.

Il fallait aussi montrer comment la cellule produit les diverses fonctions vitales et les diverses espèces vivantes, donc formuler des lois qui ne peuvent être obtenues exclusivement par simple induction. Schwann a suppléé aux lacunes de l'analyse empirique par diverses constructions théoriques : hypothèse d'une force d'attraction sélective dont l'action aboutit à la construction de la cellule; principe méthodologique voulant que la construction de l'organisme se ramène à l'embryogenèse, et donc hypothèse d'un mécanisme complexificateur et différenciateur, incarné dans l'embryon primitif, pour expliquer la formation de composés ou de dérivés des cellules; analogies de la formation blastémique et de la formation cristalline pour expliquer l'activité plastique et métabolique des cellules; enfin, principe méthodologique voulant que la synthèse de la cellule, en d'autres termes la compréhension de sa construction et de son fonctionnement, est dérivable de l'analyse, c'est-à-dire qu'elle n'est que la résultante des éléments et mécanismes qui s'y trouvent. Tout ceci délimite évidemment les voies de recherche acceptables, de même que le type de conclusions acceptables: la cellule devra avoir une structure dont il est 
plausible de dire qu'elle résulte exclusivement de causalités physico-chimiques. Tout ajout théorique dépassant l'analyse, et non effectivement dérivé d'elle, doit en être dérivable en principe et constitue une synthèse provisoire, en même temps qu'une explication partielle et provisoire des faits connus, et un programme-guide pour les recherches empiriques à venir. L'analyse empirique joue ici un rôle premier, les hypothèses n'étant recevables que si elles sont compatibles avec l'ensemble des faits admis.

Müller estime aussi qu'il faut suppléer aux lacunes de l'analyse empirique, mais l'élément suppléant doit selon lui être posé dès le début, préalablement à l'analyse: l'hypothèse de l'organisme architectonique présent sous forme de projet ou d'idée synthétique dans la manière initiale, guide la recherche empirique en lui traçant les voies de recherche acceptables. Présente dès le début sous une forme virtuelle, la synthèse précédait et déterminait l'analyse.

Le choix entre ces deux approches amène certaines conséquences pour un programme de recherche. Chez Schwann, le schéma théorique de la cellule vise une connaissance toujours plus raffinée des mécanismes, éléments et lois en cause dans le vivant, en permettant d'identifier les zones d'ignorance, et en suggérant soit de nouvelles méthodes d'analyse des faits connus, soit l'existence de faits encore non observés ainsi que la mise en place de moyens pour les détecter. Il permet en outre la sélection, pour analyse, des faits les plus pertinents ou importants, et l'intégration d'apports théoriques et méthodologiques ayant fait leurs preuves dans d'autres disciplines et adaptables. Il suggère aussi sa propre réduction à des unités plus élémentaires, grâce à des méthodes déjà utilisées avec succès. Il est une synthèse provisoire, non totalement dérivée des faits connus, mais dérivable d'eux en principe (non incompatible avec eux), et un moyen plutôt qu'une fin ou une synthèse dérivée en fait des résultats de l'analyse, dont l'existence éventuelle reste d'ailleurs problématique. L'évolution de la connaissance en fonction de faits empiriques nouvellement découverts et conséquemment en fonction aussi de nouvelles théorisations plus englobantes, plus précises et plus adéquates que les précédentes, reste indéfiniment ouverte. Chez Müller au contraire, le finalisme prend l'allure d'une représentation définitive et limite davantage les voies de la recherche empirique.

Les révisions de la théorie cellulaire par Remak, Virchow et Köller comportent deux éléments essentiels : toute cellule vient d'une autre cellule, et la cellule est une unité vitale faite d'un noyau et de protoplasme. Cela met au rancart des éléments importants du schéma schwannien : l'hypothèse du blastème et la formation de la cellule par couches ou membranes concentriques autour du noyau. Mais le schéma révisé, qui fera encore d'ailleurs l'objet d'ajustements, joue toujours le rôle décrit précédemment. Il le joue même encore mieux, car il relie en un système cohérent un ensemble de faits et de lois plus varié et plus complexe qu'auparavant. Ce sont d'ailleurs les résultats de diverses analyses empiriques lancées dans diverses directions qui ont fait voir l'inadéquation du schéma et du modèle de Schwann et forcé les théoriciens à en bâtir de nouveaux. 
Peut-on alors parler d'un seul et même programme de recherche continu, plutôt que de plusieurs programmes successifs ? Assurément non, si on se reporte aux divergences de vues entre finalistes et réductionnistes, de même qu'aux mutations du schématisme et des modèles. Cependant, une idée est demeurée active et présente tout au long de l'histoire dont l'auteur nous a retracé minutieusement les étapes: celle d'une unité dont la structure générale est commune à tous les vivants, qui explique ultimement la formation et le fonctionnement des organismes, donc en un mot tous les processus vitaux dans leur diversité, et qui sert de guide pour les recherches empiriques ultérieures. L'A. termine ses remarques sur une question qui suggère une autre recherche à faire, et dont il est éminemment souhaitable qu'elle soit faite, peut-être par l'A. lui-même. Qu'est-il advenu de la théorie cellulaire en rapport avec le transformisme néo-darwinien, la génétique post-mendélienne, et la biologie moléculaire? Ces théories ont certainement eu rapport avec la représentation de la cellule, et la question est de savoir si elles lui ont fait subir des mutations, et lesquelles.

Il y a dans ce livre un travail patient et minutieux d'analyse pour lequel il faut féliciter l'A. Il nous montre on ne peut mieux comment les voies de la recherche scientifique sont tortueuses, comment les programmes de recherche donnent souvent lieu à des résultats imprévus de leurs auteurs, comment des éléments peu compatibles peuvent coexister temporairement dans ces schémas, témoin chez Kölliker l'hypothèse cytoblastémique et le modèle de la cellule comme protoplasme nucléé se reproduisant pas scission, comment les chercheurs sont souvent forcés de reviser radicalement leurs présupposés théoriques et méthodologiques. Jusqu'à maintenant, de semblables considérations nous ont été suggérées surtout par des ouvrages d'histoire de la physique. L'histoire de la théorie cellulaire au $19^{\mathrm{e}}$ siècle ne nous présente pas de différences fondamentales sur le sujet, sauf le fait déjà connu que les théories biologiques se prêtent moins bien à la dérivation logique de faits à partir des théories et par conséquent à la prévision précise d'événements futurs, notamment dans le cas d'événements dont aucune instance analogue ou semblable n'a encore été constatée. Il faut ajouter, ce qui n'est probablement pas sans lien avec ce que nous venons de dire, que les théories physiques, plus formalisées et faisant face à une moins grande variété de phénomènes concrets, satisfont mieux à la définition lakatosienne d'un programme de recherche. Mais encore faudrait-il analyser l'histoire de certaines théories physiques, la théorie atomique en particulier, pour en constater les mutations et voir quelles analogies elles ont en ce sens avec la théorie cellulaire.

L'histoire de la théorie cellulaire au 19e siècle nous montre aussi la mise en place, inchoative et pleine de tâtonnements, à partir de réflexions sporadiques et éparpillées des chercheurs sur leur pratique, d'éléments de ce que nous appelons aujourd'hui la méthode hypothético-déductive. Elle n'est systématiquement formulée par aucun chercheur, et ne semble pas non plus, 
chez aucun d'entre eux, servir de guide systématiquement à chaque étape de la recherche. Mais on en trouve des bribes importantes, surtout chez les réductionnistes: primauté de l'analyse expérimentale; synthèses dérivables des résultats de la première, provisoires, sans prétention à une capacité explicative totale et définitive, sujettes à révision quand elles s'avèrent incompatibles avec les faits, et guides de la recherche empirique future.

Cette histoire nous montre aussi l'incontestable supériorité du réductionnisme sur le téléologisme en termes de fécondité aussi bien expérimentale que théorique. Ce sont les réductionnistes qui, partant de principes et constructions théoriques moins intouchables et fermant moins de voies à la recherche, ont fait progresser l'analyse expérimentale et la découverce de détails factuels dont la prolifération les a forcés à modifier ces mêmes constructions et principes. Ceci ne prouve en rien la fausseté des hypothèses téléologiques, mais la preuve de leur vérité reste le fardeau de ceux qui s'en font les promoteurs

On ne saurait voir dans la théorie cellulaire un paradigme kuhnien. Mais il nous apparaît qu'un tel paradigme lui est sous-jacent, soit le paradigme mécanico-atomiste devenu courant depuis Descartes et Newton. Ce paradigme dit d'abord que le monde est atomique, i.e, fait de particules relativement indivisibles et irréductibles, c'est-à-dire qui ne peuvent être divisées sans que disparaissent leurs propriétés et fonctions essentielles ; ensuite que tous les mouvements ont une cause mécanique, i.e. sont provoqués par le contact ou le choc de ces particules ou d'objets qui en sont composés, conformément à des lois déterminées. Les diverses versions de la théorie cellulaire que nous présente l'auteur satisfont toutes à ce paradigme, même celles de Müller et von Baer, qui le remettent en question seulement dans la mesure où il s'affirme comme explication suffisante et exhaustive des phénomènes vitaux : ils le jugent insuffisant et lui ajoutent l'intervention d'une force vitale. On peut affirmer que la théorie cellulaire est l'application de ce paradigme à la biologie. Mais cette application n'est pas simple et ne va pas de soi ; elle doit tenir compte de données expérimentales précises et propres au vivant, qui ne se révèlent que progressivement au cours des années. La théorie cellulaire doit forcément comporter des détails que ne comporte pas le paradigme fondamental, et peut prendre des formes diverses. Comme l'a bien vu l'A., s'en tenir au seul paradigme ne permet pas de suivre dans le détail les aménagements que lui font subir divers chercheurs en fonction de leurs croyances idéologiques, de faits expérimentaux nouvellement découverts, ou simplement de leurs intérêts de recherche spécifiques. Le programme de recherche consistait justement à montrer que le paradigme s'applique effectivement au monde vivant, et cela ne pouvait se faire sans montrer très précisément comment il s'y applique. Il fallait pour cela montrer en quoi consiste la structure de la cellule, puis comment elle est, grâce à cette structure, l'unité de base de la vie, le responsable premier de toutes les activités vitales.

Département de sciences humaines

Faculté des lettres et des sciences humaines

Université de Sherbrooke 